\title{
Title: Variation in Hospital Admission from the Emergency Department for Children with Cancer; A Pediatric Health Information System Study
}

Emily L Mueller, M.D., M.Sc ${ }^{1,2}$

Seethal A Jacob, M.D., M.S. ${ }^{1,2}$

Anneli Cochrane, M.P.H. ${ }^{1,2}$

Aaron E. Carroll, M.D., M.S. ${ }^{2,3}$

William E. Bennett, Jr., M.D., M.S. ${ }^{2,3}$

1. Section of Pediatric Hematology Oncology, Department of Pediatrics, Indiana University, Indianapolis, IN 46202

2. Center for Pediatric and Adolescent Comparative Effectiveness Research, Indiana University, Indianapolis, IN 46202

3. Section of Pediatric Gastroenterology, Hepatology, and Nutrition, Department of Pediatrics, Indiana University School of Medicine

Correspondence:

Emily L Mueller, MD, MSc

705 Riley Hospital Dr, ROC 4340

Indianapolis, IN 46202

Cell: 312-399-0245 Fax: 317-321-0128

elmuelle@iu.edu

Abstract word count: 235

Main text word count: 2445

Number of tables: 1

Number of figures: 3

Short Title: PHIS Peds Cancer ED Admission Hosp Variation

Key words: Child, Adolescent, Oncology, Supportive Care, Febrile Neutropenia

Abbreviations:

\begin{tabular}{|l|l|}
\hline ED & Emergency department \\
FN & Febrile neutropenia \\
ID & Index of Dispersion \\
ICD-9-CM & $\begin{array}{l}\text { International Classification of Diseases, Ninth Edition, Clinical } \\
\text { Modification }\end{array}$ \\
PHIS & \begin{tabular}{l} 
Pediatric Health Information System \\
\hline
\end{tabular}
\end{tabular}

This is the author's manuscript of the article published in final edited form as:

Mueller, E. L., Jacob, S. A., Cochrane, A. R., Carroll, A. E., \& Bennett, W. E. (2020). Variation in hospital admission from the emergency department for children with cancer: A Pediatric Health Information System study. Pediatric Blood \& Cancer, 67(6), e28140.

https://doi.org/10.1002/pbc.28140 


\begin{abstract}
Background: Children with cancer experience a wide range of conditions that require urgent evaluation in the emergency department (ED), yet variation in admission rates is poorly documented.
\end{abstract}

Procedure: We performed a retrospective cohort study using the Pediatric Health Information System of ED encounters by children with cancer between July 2012 and June 2015. We compared demographics for admitted versus discharged using univariate statistics, and calculated admission rates by hospital, diagnosis, day of the week, and weekend versus weekday. We assessed the degree of inter-hospital admission rates using the index of dispersion (ID).

Results: Children with cancer had 60,054 ED encounters at 37 hospitals. Overall, 62.5\% were admitted (range 43.2-92.1\%). Children with cancer that visited the ED for a primary diagnosis of fever experienced the largest amount of variability in admission with rates, range $10.4 \%$ to $74.1 \%$ (ID of 8.1). Less variability existed among hospital admission rates for both neutropenia (range $60-100 \%$, ID 1.0) and FN (range 66.7-100\%, ID 0.83), when compared to fever only (range 10.4-74.1\%, ID 8.1). Admission rates by day of the week did not demonstrate significant variability for any of the scenarios examined (overall $\mathrm{p}=0.91$ ). There were no differences by weekend versus weekday either (overall $p=0.52$ ).

Conclusion: The percentage of children with cancer admitted through the ED vary widely by institution and diagnosis. Standardization of best practices for children with cancer admitted through the ED should be an area of continued improvement. 


\section{Background}

Children with cancer experience a wide range of symptoms and complications that require urgent evaluation. ${ }^{1,2}$ While some pediatric oncology clinics have the capacity to evaluate patients during the weekdays, many children with cancer are evaluated and treated in the emergency department (ED) based on patient distance from clinic, clinic resources, and patient severity. The ED is also the primary avenue for evaluation and admission to the hospital during non-business hours and weekends. ED admission rates have been explored in other pediatric conditions including neonatal fevers and gastroenteritis with demonstration of significant inter-hospital variation in management and admission rates. ${ }^{3,4}$ In spite of this, variation has not been explored among children with cancer.

Variation for this population should be explored due to unique features related to ED utilization by children with cancer. Previous studies of ED utilization among children with cancer have revealed that this unique population comprises a small proportion of all visits to the ED, ${ }^{1,2}$ which may lead to less familiarity among ED providers. There are also differences in provider practices and inconsistent recommendations for those patients who live further away from their treating institution. ${ }^{5}$

There are no studies specifically describing the variation in admission rates from the ED for children with cancer. Therefore, the objective of this study was to determine interhospital variation in admission rates for children with cancer who present to the ED, 
stratified by presenting complaint and day of the week. Our hypothesis is that variation exists among hospitals and that the variation would be greatest during the weekdays.

\section{Methods}

Study Design and Data Source

We conducted a retrospective cohort study using the Pediatric Health Information System (PHIS), an administrative and resource utilization database from 49 children's hospitals in non-competing markets. All hospitals are affiliated with the Children's Hospital Association (CHA, Shawnee Mission, KS) and are in 26 states plus the District of Columbia. They account for $\sim 20 \%$ of all pediatric hospitalizations in the United States. ${ }^{6}$ Hospitals were included in this study if they provided consistent inpatient and ED data between July 1, 2012, and June 30, 2015, resulting in 37 hospitals included. The Children's Hospital Association (Overland Park, KS) and participating hospitals jointly monitor both the quality and integrity of data, described previously. ${ }^{7}$ The data warehouse function for the PHIS database is managed by Thomson Reuters (Ann Arbor, MI). Participating hospitals provide de-identified patient discharge/encounter data including demographics, billing data, diagnoses, and procedures. This analysis was based on deidentified data and was therefore considered exempt from institutional review board approval by Indiana University School of Medicine. Identifying hospital numbers were anonymized for the study analysis.

\section{Study Population/Identification of Cases}


ED encounters by children with cancer were identified by the inclusion of an International Classification of Diseases, Ninth Edition, Clinical Modification (ICD-9CM) code for a malignancy (140.x-209.x, 235.x-239.x), as previously described. ${ }^{1,2} \mathrm{We}$ chose not to use ICD-10 data, as there are inconsistencies across diagnoses. An ED encounter was defined as either an inpatient or observation encounter with an associated ED charge flag (i.e. admission) or an ED encounter with discharge to home (i.e.

discharge). We also determined the following demographic variables for each subject: gender, race, ethnicity, insurance type, and age. Age in years was transformed into a categorical variable as follows: Ages 0-4, 5-9, 10-14, and 15 and older. Encounters for patients older than age 25 were excluded. Type of insurance was categorized as public, private, and other. Race and ethnicity variables were combined as: Non-Hispanic white, Non-Hispanic black, Hispanic, Asian, and other/unknown.

\section{Exploratory variables}

In order to explore variation among ED admissions, we chose to evaluate the admission rates in several different ways. Since fever, neutropenia, and febrile neutropenia are the most common reasons for ED visits among children with cancer, ${ }^{1,2}$ we made variables for each of these conditions. The primary diagnosis for the ED visit was defined as the first ICD-9-CM discharge diagnosis, unless the first diagnosis was a cancer diagnosis. In cases where a cancer diagnosis was the first diagnosis, the second listed ICD-9-CM diagnosis was considered to be the reason prompting the ED visit. Cancer diagnoses were not considered the reason prompting an ED visit because we intended to focus on symptoms or complications associated with ED visits made by children with cancer. A primary 
diagnosis of fever was defined as an ICD-9-CM discharge diagnosis of 780.6, 780.60 or 780.61. A primary diagnosis of neutropenia was defined as an ICD-9-CM discharge diagnosis of $288.0,288.00,288.03,288.04$ or 288.09 . Given that the presence of febrile neutropenia (FN) has clinical importance but does not have a unique ICD-9-CM code, we combined codes for fever and neutropenia as previously published. ${ }^{2}$ Among patients with a primary diagnosis of fever, all associated diagnoses were evaluated for the presence of neutropenia and vice versa. Then, an encounter was labeled as FN if their primary diagnosis was fever with neutropenia in any of the diagnostic fields or primary diagnosis of neutropenia with fever in any of the diagnostic fields.

\section{Statistical Analyses}

We compared subject demographics for those who were admitted versus discharged using univariate analysis with the chi-square test, since all of our variables were categorical variables. Hospital-level admission rates were calculated for a series of scenarios. First, we calculated overall admission rates by hospital for the entire time period, then by day of the week. Admission rates for a primary diagnosis of fever, neutropenia, and the FN variable were calculated both overall and by day of the week.

The degree of inter-hospital variation in admission rates for each of the specific scenarios listed above were evaluated using a common statistical test called the index of dispersion (ID), which represents an estimate of the overall randomness of a sample, and is useful when comparing percentages (e.g. admission rates) across multiple institutions. This measure was chosen to assess variation because it is dimensionless, and can be less 
sensitive to a small number of outliers. ID values greater than 1 indicate data that is overdispersed (e.g. greater variability). Hospitals who had a total number of encounters that were less than five for a specific diagnosis or day of the week were excluded from that calculation to avoid a skewed variability measurement based on a small N.

Finally, we performed a one-way ANOVA to compare variation by hospital, by diagnosis (fever, neutropenia, and FN diagnoses), and by day of the week. We compared weekend (Saturday and Sunday) versus weekday using Student's t-test. All analyses were performed using SAS v9.4 (SAS Institute, Cary, NC) and Microsoft Excel.

\section{Results}

We identified 60,054 ED encounters for children with cancer at 37 hospitals during the three-year study period. Of those encounters, $62.5 \%$ were admitted to the hospital, and $37.5 \%$ were discharged home from the ED. The study population (shown in Table 1) was mostly male (54.2\%) and the most common race/ethnicity was non-Hispanic white (47.2\%). The majority of patients had public insurance $(54.8 \%)$, followed by private (40.4\%), and other (4.7\%). There were more younger children with $33.2 \%$ age 4 years or under and $27.2 \%$ were between ages 5 and 9 years. There were statistically significant differences in all demographics between admitted and discharged, which was explored in a previous publication. ${ }^{1}$

In the evaluation of overall variation in hospital admission rates, admission rates ranged from 43.2 to $92.1 \%$, with a median of $63.7 \%$ (Figure 1 ). For overall admission rates by 
hospital, the index of dispersion (ID) was $2.6(\mathrm{~N}=37)$, indicating over-dispersed data with high variability.

On further evaluation by primary diagnosis of fever or neutropenia, differences in variation persisted (Figure $2 \mathrm{~A}$ and $\mathrm{B}$ ). Children with cancer that visited the ED for a primary diagnosis of fever experienced the greatest amount of variability in admission rates ranging from $10.4 \%$ to $74.1 \%$ (median $33.8 \%$ ), see Figure $2 \mathrm{~A}$. For fever diagnosis, we calculated an ID of 8.1 ( $\mathrm{N}=37$ hospitals), indicating a large dispersion in this measure. Admission rates for a primary diagnosis of neutropenia ranged from 60 to $100 \%$ (median 91.7\%), see Figure 2B. For neutropenia diagnosis, we calculated an ID of 1.0 ( $\mathrm{N}=36$ hospitals). Finally, when evaluating encounters that had a diagnosis of FN, admission rates ranged from 66.7 to $100 \%$ (median 94.5\%), see Figure 3. For FN, we calculated an ID of $0.83(\mathrm{~N}=36)$. These results indicate a high degree of variability for fever alone, but markedly less dispersion of data with neutropenia alone or when both fever and neutropenia was present.

When comparing hospital rates between institutions, we found no statistically significant effect of day of the week on variation for overall admission rates $(p=0.91)$. For each of the sub analyses, we found no difference among hospitals by day of the week for fever $(p=0.80)$, neutropenia $(p=0.49)$, or $F N(p=0.73)$. There were also no differences found when comparing weekend versus weekday for overall admission rates $(p=0.52)$, fever $(p=0.58)$, neutropenia $(p=0.93)$, and $F N(p=0.10)$ 


\section{Discussion}

In this retrospective cohort study using administrative data from 37 US children's hospitals, we found wide variability in admission rates between hospitals for children with cancer who sought care in the ED. Previous literature has demonstrated that there was variation in admission rates by reason for ED visit, ${ }^{1,2}$ and we have now shown that there is also variation by hospital for each of the scenarios investigated. The greatest variability was for a primary diagnosis of fever, with less variability among a primary diagnosis of neutropenia or FN. Day of the week or weekend versus weekday did not have a significant impact on between hospital variation.

We found the highest amount of variability for ED admission rates overall and for ED visits with a primary diagnosis of fever. There was less variability when the primary diagnosis was neutropenia or if the patient met the criteria to be labeled as FN. Interestingly, there was no significant impact on admission rates for this population in any of the clinical scenarios when evaluating by day of the week or weekend vs weekday. These findings highlight several important areas for future investigation. For the variation in admissions by hospital seen for the primary diagnosis of fever, patient level information is needed to better understand the circumstances surrounding the ED visits. Specifically, evaluating if there was an objective fever documented and the impact of their laboratory values including their absolute neutrophil count. The lack of effect by day of the week was a surprising finding, and thus deserves future investigation to understand the role of communication and triage decision-making that occurs between 
ED providers and oncologists when children with cancer present to the ED, and how it may differ from other conditions.

When considering the variation for overall admission rates by hospital, the causes are likely multi-factorial and include both hospital-system, provider-level, and patient-level contributions. Some of the variation may be due to differences in triaging practices among institutions and also among providers within the same institution. Some institutions routinely send all children with cancer to the ED for management, while others have the capacity to evaluate and manage urgent clinical needs in the pediatric oncology clinic. From a provider-level, there are differences in preferences and practices specifically around the treatment of fevers in pediatric oncology patients, ${ }^{5}$ which may influence ED admission rates.

When an ED visit had a primary diagnosis of fever with any associated diagnosis of neutropenia, the variation in admission rates went down significantly and the median admission rates were much higher. These findings appear to suggest that the general clinical expectations is that children with cancer who present to the ED with febrile neutropenia are treated similarly with upfront inpatient admission for treatment and monitoring. ${ }^{8,9}$ Yet interestingly, there continues to be a lack of a clear consensus among pediatric oncologists on the management of $\mathrm{FN}$, despite continued evidence that there are risk stratification schemas that can be instituted. ${ }^{10-15}$ 
Previous analyses show differences in admission rates and outcomes for patients who seek care in the ED during the weekends. ${ }^{16-23}$ Yet this was not found to be a significant factor in admission rates for children with cancer. Since ED encounters by children with cancer are a very small proportion of national ED visits, ${ }^{2}$ ED providers are likely not as familiar with their care and management, thus oncology providers routinely are consulted by phone to discuss each patient who presents to the ED. An important research avenue to explore would be to evaluate and quantify the amount of variation by oncology providers both within and between hospital systems. Additionally, future research could focus on qualitative differences among ED providers' views of shared patient care for children with cancer, which could determine the ways in which clinical decisions are made and how to optimize those points of communication.

\section{Limitations}

While this study provided insight into admission rate variation among hospitals, several important limitations should be considered. As with previous analyses of PHIS data, we used ICD-9-CM codes to capture our patient population and evaluate the reasons for their ED encounter. Therefore, it is possible we are not accurately identifying all patients with cancer if their diagnosis was not included on an encounter, which could lead to over or under estimation of ED cases. PHIS dataset does not reliably determine the chief complaint that led to the ED visit, so the reason for the ED visit was extrapolated from ICD-9-CM codes. PHIS does not include all hospitals that diagnose and treat children with cancer, thus it was not possible to accurately determine if a subject presented to the ED for a new diagnosis or continued care. We chose not to include ICD-10 data in order 
to decrease the chance for inconsistencies with conversion. We also did not have access to important clinical data such as vital signs and physical exam findings, laboratory results, or imaging results which impact clinical decision making. We attempted to evaluate for differences by day of the week, but our evaluation does not account for holidays which may impact hospital resources, such as staffing patterns, that could affect decision making. For this evaluation, we also did not intend to address the necessity or preventability of the hospital admission since we do not have access to key clinical features, severity of illness, or sociodemographic factors that may have impacted clinical decision making.

\section{Conclusion}

In conclusion, we found that the percentage of children with cancer admitted through the ED varies widely by institution and by diagnosis. When evaluated by diagnosis, patients who presented with fever had the highest amount of variation in admission rates. Further research is needed to better understand the sources of the variation including patient level clinical presentation details, provider preferences, or institutional policies that lead to the greatest amount of variability. Next steps would then be to use this information to create standards of best practices for children with cancer who are evaluated in the ED.

Funding Source A portion of Anneli Cochrane's salary was provided by the Jeff Gordon Children's Foundation.

Financial Disclosure The authors have no financial disclosures

Conflict of Interest The authors have no conflicts of interest to disclose 
Author Contributions All authors affirm that we have participated in the concept, drafting, and revising of the manuscript. We have all approved the manuscript in its current final format for submission.

Acknowledgements None 


\section{References}

1. Mueller EL, Hall M, Shah SS, August KJ, Radhi MA, Macy ML. Characteristics of children with cancer discharged or admitted from the emergency department. Pediatr Blood Cancer. 2016;63(5):853-858.

2. Mueller EL, Sabbatini A, Gebremariam A, Mody R, Sung L, Macy ML. Why pediatric patients with cancer visit the emergency department: United States, 2006-2010. Pediatr Blood Cancer. 2015;62(3):490-495.

3. Aronson PL, Thurm C, Alpern ER, et al. Variation in care of the febrile young infant $<90$ days in US pediatric emergency departments. Pediatrics. 2014;134(4):667-677.

4. Jain S. Elon LK, Johnson BA, Frank G, DeGuzman M. physician practice variation in the pediatric emergency department and its impact on resource use and quality of care. Pediatr Emerg Care. 2010; 26(12);902-909.

5. Mueller EL, Walkovich KJ, Yanik GA, Clark SJ. Variation in Management of Fever and Neutropenia Among Pediatric Patients With Cancer: A Survey of Providers in Michigan. Pediatr Hematol Oncol. 2015;32(5):331-40.

6. Colvin JD, Hall M, Berry JG, et al. Financial Loss for Inpatient Care of MedicaidInsured Children. JAMA Pediatr. 2016;170(11):1055-1062.

7. Mongelluzzo J, Mohamad Z, Ten Have TR, Shah SS. Corticosteroids and mortality in children with bacterial meningitis. JAMA. 2008;299(17):2048-2055.

8. Freifeld AG, Bow EJ, Sepkowitz KA, et al. Clinical practice guideline for the use of antimicrobial agents in neutropenic patients with cancer: 2010 update by the infectious diseases society of america. Clin Infect Dis. 2011;52(4):e56-93.

9. Pizzo PA. Management of fever in patients with cancer and treatment-induced neutropenia. The New England journal of medicine. 1993;328(18):1323-1332.

10. Henry M, Sung L. Supportive Care in Pediatric Oncology: Oncologic Emergencies and Management of Fever and Neutropenia. Pediatr Clin North Am. 2015;62(1):27-46.

11. Lehrnbecher T, Robinson P, Fisher B, et al. Guideline for the Management of Fever and Neutropenia in Children With Cancer and Hematopoietic Stem-Cell Transplantation Recipients: 2017 Update. J Clin Oncol. 2017;35(18):2082-2094.

12. Manji A, Beyene J, Dupuis LL, Phillips R, Lehrnbecher T, Sung L. Outpatient and oral antibiotic management of low-risk febrile neutropenia are effective in children--a systematic review of prospective trials. Support Care Cancer. 2012;20(6):1135-1145.

13. Paolino J MJ, Lucas A, et al. Outcomes of a clinical pathway for primary outpatient management of pediatric patients with low-risk febrile neutropenia. Pediatr Blood Cancer. 2019;66:e27679.

14. Teuffel O, Ethier MC, Alibhai SM, Beyene J, Sung L. Outpatient management of cancer patients with febrile neutropenia: a systematic review and meta-analysis. Ann Oncology. 2011;22(11):2358-2365.

15. Vidal, L Ben dor I, Paul M, Pokroy E, Soares-Weiser K, Leibovici L. Oral versus intravenous antibiotic treatement for febrile neutropenia. Cochrane Database Syst Rev. 2013;10:CD003992. 
16. Thompson RT, Bennett WE, Jr., Finnell SM, Downs SM, Carroll AE. Increased length of stay and costs associated with weekend admissions for failure to thrive. Pediatrics. 2013;131(3):e805-810.

17. Kostis WJ, Demissie K, Marcella SW, et al. Weekend versus weekday admission and mortality from myocardial infarction. NEJM. 2007;356(11):1099-1109.

18. Saposnik G BA, Bayer N, Hachinski V. Weekends: a dangerous time for having a stroke? Stroke. 2007 Apr;38(4):1211-5.

19. Ananthakrishnan AN ME, Saeian K. Outcomes of weekend admissions for upper gastrointestinal hemorrhage: a nationwide analysis. Clin Gastroenterol Hepatol. 2009 Mar;7(3):296-302e1.

20. Aujesky D JD, Mor MK, Geng M, Fine MJ, Ibrahim SA. Weekend versus weekday admission and mortality after acute pulmonary embolism. Circulation. 2009 Feb 24;119(7):962-8

21. Crowley RW YH, Stukenborg GJ, Ionescu AA, Kassell NF, Dumont AS. Influence of weekend versus weekday hospital admission on mortality following subarachnoid hemorrhage. Clinical article. J Neurosurg. 2009 Jul;111(1):60-6

22. Crowley RW YH, Stukenborg GJ, Medel R, Kassell NF, Dumont AS. Influence of weekend hospital admission on short-term mortality after intracerebral hemorrhage. Stroke. 2009 Jul;40(7):2387-92

23. Barba R ZA, Losa JE, Marco J, Plaza S, Rosado C, Canora J. The impact of weekends on outcome for acute exacerbations of COPD. Eur Respir J. 2012 Jan;39(1):46-50. 


\section{Figures}

FIGURE 1. Overall Emergency Department Admission Rates for Children with Cancer by Hospital

FIGURE 2. Emergency Department Admission Rates for Children with Cancer by Primary Diagnosis of A) Fever and B) Neutropenia, by Hospital

FIGURE 3. Emergency Department Admission Rates for Children with Cancer by Diagnosis of Febrile Neutropenia, by Hospital 